\title{
Increasing Incidence of Hypertension in Females by Nasira, Maryam, Salman, and Rehan (2019)
}

\author{
Alqahtani Ibtesam Mohammed \\ King Saud University, School of Nursing, Riyadh 11451, Saudi Arabia \\ Email: Ibtesam.alqahtani@mymail.barry.edu
}

Dear Editor,

I am writing to respond to an article that was written by Nasira Tajamal, Maryam Tajamal, Salman Tajammal, and Rehan Tajammal, entitled "Increasing Incidence of Hypertension in Females," published May 01, 2019. It is significant to bringing healthcare providers' attention to an underestimated and undiagnosed health issue that occurs among women, which is hypertension. Hypertension is one of the cardio-metabolic risk factors that is associated with the increased burden of morbidity and mortality globally. As stated in this article, over the last two decades the prevalence of hypertension has increased. In 2000 the United Nations reported that the estimated total number of adults with hypertension was 972 million, and this number is predicted to increase to reach 1.56 billion by $2025 .^{[1]}$

Hypertension is a chronic condition characterized by elevated blood pressure in the arteries, which leads to increased effort of the heart more than normal in order to circulate blood through the blood vessels. ${ }^{[2]}$ This disease is a silent threat to all individuals' health throughout the world. Evidence shows that 9.4 million individuals die yearly due to complications of hypertension, and 1.58 billion adults will suffer from hypertension complications by $2025 .{ }^{[3]}$ Hence, early detection and management of this disease is significant.

\section{Discussion}

As stated in this article, the major risk factor for cardiovascular disease (CVD) is hypertension. Women are at greater risk of developing CVD, which is considered a primary leading cause of death than any other disease. Evidence shows that one in every five American women die because of high blood pressure. ${ }^{[4]}$ Unfortunately, women have unique risk factors that are needed to take into consideration to help prevent hypertension-related morbidity and mortality. As mentioned in this article, the elevated blood pressure among women is attributable to various factors such as physical inactivity, poor diet quality, obesity, aging, stress, oral contraceptive pills, pregnancy, and post menopause.

With the aging of the population, lifestyle changes, stress, and obesity, the susceptibility of hypertension on women increases. Most importantly, there is an urgent need for implementing more evidence-based prevention and management measures in health care services. Establishing an action plan based on evidence to identify high-risk women in an early stage is vital. Early detection and screening in early adulthood may offer a window of opportunity to improve women's health outcomes. Increasing women's education about the risk factors that are unique to them and encouraging them to adopt healthy lifestyles, particularly exercise, are other significant actions to forestall the burden of hypertension. Increasing women's awareness about good lifestyle habits empower them to take control of their health and become partners with healthcare providers to control the problem. Special attention should be paid to overweight women. Weight reduction is beneficial in hypertensive women and should be encouraged.

\section{Conclusion}

This article indicates the need for further investigation of hypertension prevalence, treatment, and control among women in Asian countries. Future research that identifies barriers that contribute to adopting healthy lifestyles among hypertensive women in Asian countries are required. Such research is essential to developing effective awareness programs that would help women in those countries to overcome these barriers and adopting healthy lifestyles to control the epidemic of hypertension.

\section{References}

[1] Carba, D., Bas, I., Gultiano, S., Lee, N., \& Adair, L. Waist circumference and the risk of hypertension and prediabetes among Filipino women. European Journal of Nutrition vol. 52, 2 (2013): 825-832. https://doi.org/10.1007/s00394-012-0390-9

[2] Carey, R. M., \& Whelton, P. K. (2018). Prevention, Detection, Evaluation, and Management of High Blood Pressure in Adults: Synopsis of the 2017 American College of Cardiology/American Heart Association Hypertension Guideline. Annals of Internal Medicine vol 168, 5 (2018): 351-358. https://doi.org/10.7326/M173203

[3] Khader, Y., Batieha, A., Jaddou, H., Rawashdeh, S. I., El-Khateeb, M., Hyassat, D., Ajlouni, K. Hypertension in Jordan: Prevalence, Awareness, Control, and Its Associated Factors. International Journal of $\begin{array}{llll}\text { Hypertension } & \text { vol } 2019 & \text { (2019): } & 1-8 .\end{array}$ https://doi.org/10.1155/2019/3210617

[4] Wenger, N. K., Arnold, A., Bairey Merz, C. N., CooperDeHoff, R. M., Ferdinand, K. C., Fleg, J. L., Pepine, C. 
J. (2018). Hypertension Across a Woman's Life Cycle. Journal of the American College of Cardiology (JACC) vol $\quad 71, \quad 16 \quad$ (2018):1797-1813. https://doi.org/10.1016/j.jacc.2018.02.033

\section{Author Profile}

Alqahtani Ibtesam received a B.S. degree in Nursing from King Saud University in the Kingdom of Saudi Arabia, and received an M.S degree in Nursing Education from Roberts Wesleyan College in the United States of America respectively. Currently, she is completing her PhD in Nursing at Barry University in the state of Florida, the United States of America. 